\title{
Communication between Physics and Society
}

\section{G. Diemer, Eindhoven}

This paper deals with the responsibility of EPS and its members to stimulate communication between the physics community and society-atlarge. Based on personal experience, the author concludes that an important aspect is the improvement of the information mechanism, and he emphasizes the crucial role of the management of institutes, organizations and private enterprises that employ physicists. When management takes the initiative in improving the communication channels and creates adequate organization structures for this, both society as a whole and the institute or enterprise itself may profit. Long-run results might be that society and physics do not drift further apart as regards goals and attitudes and that science policy and planning improve.
For several years now it has been my job to stimulate publicity on research news from a large international science-based industrial company. I thought it worthwhile to draw some conclusions from my experience that may be of interest in those EPS circles that aim at improving the relation between physics and society.

However, realizing that the situation varies strongly from country to country, I have tried to avoid single sidedness, as much as was possible, by presenting a rather abstract generalisation of my personal experience.

The relation between physics and society is a matter of communication and interaction and I should like to interpret these notions in their widest sense, that is, really as mutual influence. Furthermore, I should like to

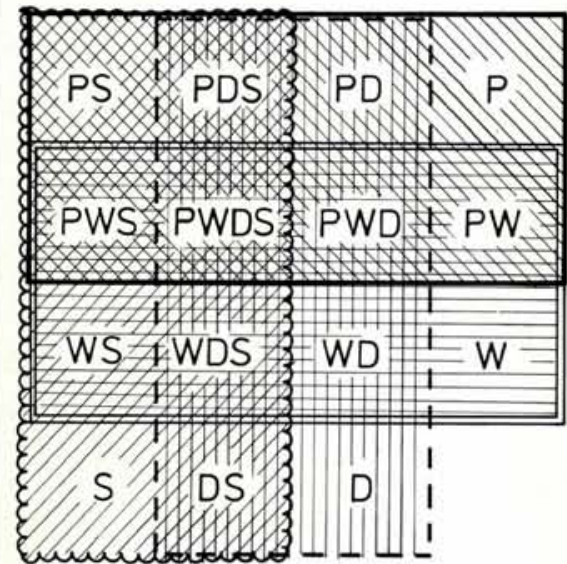

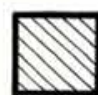

$P$ (personal)
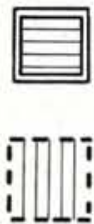

D (discipline)

S (society at large) stress the complexity of concepts such as 'physics' and 'society'. In order to obtain a framework for the discussion let us distinguish between the following areas

$P$ : the personal, private life of the physicist ;

$W$ : the institute, organization or enterprise in which the physicist carries out his professional activities and which is the scene of his daily work;

$D$ : the discipline of physics, that is, the world-wide 'invisible college', according to Ziman.

$S$ : society, that is, the structured ensemble of all human beings.

Each of these areas has a certain overlap with others and in the 11 overlapping areas (indicated by $P W, P W D$, $P W D S$, etc.) different types of interaction take place (see diagram).

The type and intensity of these interactions depend on the specific interests, internal structure and functioning, and power of each of the areas $P, W, D$, and $S$. No detailed analysis and evaluation of all of the interaction areas is available and I want to comment only on some.

Things like motivations, beliefs, interests, will, aims, morale, expectations and rationale determine human behaviour. Small groups, in which personal interactions can be strong, may play an important rôle in the interactions, especially when they

\section{Contents}

Communication . . . . . 1

Research, Science and

Education . . . . . 3

Interactive Data System . . 4

Danish Physical Society . . 5

Ionized Gases . . . . . . . 6

Atomic Spectroscopy . . . 7

Pollution in the Air . . . . 7

Meetings . . . . . . . 8 
operate at an intermediate level of hierarchy. They then act as links, both vertically in internal interaction via hierarchical lines (downwards and upwards) and, horizontally, in external communication.

Areas $P$ and $W$ bring with them strong motive power, of an existential nature. Their interactions with the rest of the system are vital and are felt in daily life. $W$ can, much more than $P$ and $D$, intensify interactions with $S$, if desired. The fact that EPS acts mainly through $D$, whose power of direct interaction with $S$, is much less than that of $W$, may serve to warn us not to overestimate the effect of EPS action in this field. Stimulation through $D$, to form linking groups as mentioned above, may however, have a non-negligible effect on the interactions wanted.

As far as the form of communication is concerned, I uphold the thesis that, with respect to efficiency, the traditional operations of printing and reading still rank high, on the condition that, of course, content and form are well adapted to the reader. (Appendix I presents, in a light-hearted way, some arguments for this statement.) On the other hand, audio-visual media, like film and TV, are gaining momentum, especially where one aims at improvement of interactions in the affective sphere. Scientists are, in general, not yet well-accustomed to this form of communication.

Let us look at a few of the stronger and weaker points in some of the overlapping areas, points that are of predominant interest as regards the actual situation in society-at-large and at the role of physics.

In the $(P D)$ and (WD) areas of overlap, intense, frequent and quite adequate communication patterns exist (the 'invisible college'). One could speak here of a valuable inheritance from the past which, however, has led to $P$-attitudes and $P$-abilities which sometimes may hamper the improvement of various types of communication (PS, WS, DS, PDS, WDS) with society-at-large (for example, esoteric or specialist language).

The (WD) interaction, even for private enterprises, is also rather strong. $W$ gains from it, provided that $W$ 's proper interests are safeguarded by adequate $(P W)$ communication and, where desired, by adequate patent policy.

In interactions with $S$, two different levels are of main importance, that of the subject matter and that of science policy. Because of their importance to the present and the future situation of physicists, we shall discuss the lat- ter interactions at the two levels mentioned in somewhat more detail. Here, again, for an effective interaction to be reached, the presence of an adequate organizational set-up is prerequisite.

\section{Interactions with society-at-large}

\section{(1) The subject matter}

In the (PS), (DS), (PWS), (PDS) and $(P W D S)$ relations, adequate information should be provided, to other scientists and to the public-at-large, such that political choices or decisions and actions by government can be based on adequate knowledge of the real state of affairs. At the interface, we have civil servants, politicians, science journalists, press officers and school teachers. In order to motivate $W$ to cooperate, it is advisable as a first step to increase the $(P W)$ communication in such a way that results obtained by individual workers and their implications for $W$ are presented in a condensed form. It should not require too much effort on the part of the non-specialist $W$ readers to grasp what is essential to them. An internal ( $P W)$ magazine, edited by such part-time $P$ 's in a $W$-community that are capable of providing the right form of presentation in text and illustration, can play an important role here. W-management should have a concern in such an increased internal communication. In private enterprise, this has to be synchronized with and adapted to patent policies.

In our research organization we have found about $20 \%$ of the total scientist 'output' (that is, subjects dealt with in scientific and technical reports, publications and patent applications) to be suitable for such a $(P W)$ magazine, and, on inquiry, it was found that it considerably increases the communication within the organization.

Apart from increased $(P P)$ and $(P W)$ contacts, it is a good basis for (PS) and (PDS) communications, which may be selected from it. Selection criteria and presentation are mainly based on what might be of interest to the $(D S)$ and the S-reader, respectively, and on what is likely to capture their attention (see Appendix 2). We selected about $3 \%$ of the total output for research press releases, to be sent to publication media and about a third of these also found their way to the daily newspapers. Reactions from readers to the scientists (whose names are mentioned in the press- releases) are several times greater than those originating from scientific publications and have been found stimulating to both the scientist and to the management of $W$.

\section{(2) Science policy}

Here, interaction in the (WS) and (WDS) areas are of prime importance. However, on the S-side corresponding organization patterns and procedures are generally weak or inefficient. Increased interaction, which is required for any decision-making on the basis of greater responsibility, is therefore difficult to achieve. I feel that intensified information at level (1) as well as increased interaction in the $(P W)$ area could help.

\section{Conclusion}

I think it the responsibility of EPS and its members to stimulate interaction between physicists and societyat-large, both as regards subject matter and science policy. In the long run, not only $S$ as a whole, but also $W$ as a part of it, will profit from an increase in such interactions.

\section{Acknowledgement}

Thanks are due to J. Volger for stimulating discussions and amendments.

\section{Appendix 1 Communication
by P.R.I.N.T.}

(freely adapted from R. J. Heathorn's "Learn with BOOK", Punch)

A new aid to rapid - almost magical - communication and information has made its appearance. Indications are that, if it catches on, all electronic gadgets will be so much junk.

The device is known as PRecise INformation Tool. The makers generally call it by the acronym PRINT.

Many advantages are claimed over the old-style information and communication aids on which most people are brought up nowadays. It has no wires, no electric circuit to break down. No connection is needed to an electricity power point. It is made entirely without mechanical parts to go wrong or need replacement. The cheap process for mass production of PRINTs makes it possible for the user to have a private, dedicated data set to hand for use at any moment. 
Anyone can use PRINT, even children, and, thanks to its ergonomic design, its handling does not cause psychical or physical stress. It fits comfortably into the hands and it can be conveniently used sitting in an an armchair by the fire. A small accessory, available at trifling extra cost, is the pencil. This enables the user to add or skip information, to adapt the system to personal needs. Unnecessary information is easily erased or discarded by throwing it into the fire.

How does this revolutionary, unbelievably easy invention work? Basically, PRINT consists only of one or more paper sheets. Each sheet bears a number in sequence, so that the sheets can be used in an adequate order. Each sheet of paper presents the user with a sequence in the form of symbols and/or images which he absorbs optically for automatic registration in the brain. When one sheet from a multisheet PRINT has been assimilated, a flick of the finger turns it over and further information is found on the next sheet.

PRINT is, in fact, a low-cost and space-saving display. If desired, both sides of each sheet may be used and in this way even greater economy is effected. No buttons need to be pressed to move from one sheet to another or to start a PRINT working. PRINT may be taken up at any time and instantly it is ready for use, since nothing has to be connected up or switched on. The user may turn at will to any sheet or to any part of a particular sheet, going backwards or forwards as he pleases. PRINT thus provides true random access to a non-volatile memory.

PRINTs may be stored in handy portfolios on the cover of which a schedule may be indicated for ease of reference. Thus a convenient method is provided for compiling private data sets instead of having access to central, on-line data sets via complicated remote terminals. PRINT also offers the possibility of simultaneous display of any number of data sheets without the need for display terminals with multiple-page facilities. A selection of most interesting information can be presented in the form of 'pictures on the wall'.

PRINT requires no upkeep costs ; no batteries or any other power supply are needed, since the motive power, thanks to an ingenious device patented by the makers, is supplied by the brain of the user.

Altogether the PRecise INformation Tool seems to have great advantages and no drawbacks. We predict a big future for it.

\section{Appendix 2 \\ Criteria for mass media communication}

For selecting the subjects:

a social relevance; applications recognizable to layman ;

b timeliness;

c elements of tension, adventure, conflict.

For form of presentation :

a use heading and illustrations as eye-catchers ;

b support acceptance of printed material by personal contacts between publicists and research institutes (e. g. once a year) ;

$c$ audio-visual media (e. g. T.V.) can be much more powerful, especially for subjects with strong affective aspects (social relevance of a research item, motivation for physics, career possibilities, science policy, etc.).

However, efficient presentation asks for a considerable effort from the research institute; therefore, only advisable on special occasions.

\section{Research, Science and Education}

Research, Science and Education is the title of the 27-page programme of work proposed by R. Dahrendorf, Commissioner responsible for these areas within the European Economic Community. In introducing the programme, Dahrendorf indicates that science and education should play a special role in bringing the economic growth of the Community to the service of mankind and the quality of life. Because he clearly realized that things are not automatically good through being done on a European scale, the Commissioner confined his working programme to three kinds of task :

(i) Measures in the fields of research, science and technical information which are prescribed as Community tasks, or which arise for Community policies in other fields ;

(ii) Measures which are in themselves likely to further European cooperation and thus support the general aims of the Community ;

(iii) Measures which can be better undertaken at Community level than at national level or at that of international organizations.

There are no special references to physics as such in the programme, but it is clear that its fulfillment will depend much on the active cooperation of European physicists. Several highlights from the programme are especially relevant to the goals of the European Physical Society:

\section{Free movment of persons}

Dahrendorf states that there should be hearings of groups and organizations concerned by freedom of establishment for professional persons, in order to make rapid progress towards the mutual recognition of diplomas. Also, educational cooperation with other countries, in particular developing countries, should include the offer of more scholarships and the provision of experts.

\section{Science}

For a common scientific policy to be devloped, according to the programme, scientific cooperation in pure research between Member States of the Community will have to be promoted and made more efficient. Some of the steps along the path towards attaining this objective are:

(i) Greater mobility of research scientists within the Community;

(ii) Greater facilities for international meetings in the Community;

(iii) Stimulation of concerted action and cooperation projects in Europe ;

(iv) Listing of laboratories which could develop into special research establishments at the European level; creation of a specialized network of such laboratories ;

(v) Coordination in costly long-term projects ;

(vi) Common use of expensive heavy equipment.

\section{Research and development}

The major part of the programme is concerned with defining $R$ \& $D$ objectives, topics, activites and organization. Dahrendorf believes that $R$ \& $D$ policy is particularly important, since Member States' willingness to (Continued on page 7) 